\title{
Homogenization of Very Thin Elastic Reticulated Structures
}

\author{
J. Casado-Diaz, M. Luna-Laynez ${ }^{1}$, J.D. Martin \\ Departamento de Ecuaciones Diferenciales y Analisis Numerico, \\ Universidad de Sevilla, c/Tarfia s/n, 41012-Sevilla, España \\ 'mllaynez@us.es
}

\section{SUMMARY}

This work is devoted to the homogenization of the anisotropic, linearized elasticity system posed on thin reticulated structures involving several parameters. We show that the result depends on the relative size of the parameters. In every case, we obtain a limit problem where both the microscopic and macroscopic scales appear together. From this problem, we get an asymptotic development which gives an approximation in $L^{2}$ of the displacements and the linearized strain tensor.

\section{INTRODUCTION}

In $/ 5 /$ and $/ 13 /$, using an original adaptation of the Arbogast $e t$ al. method /1/, we studied the asymptotic behavior of the solutions of diffusion problems posed on thin reticulated structures involving several small parameters. In this work we consider the linearized elasticity system. To simplify the exposition, we deal with a particular structure, $\Omega_{\varepsilon}$, shaped by the union of orthogonal beams, with thickness $\varepsilon d_{\varepsilon}$, disposed periodically, along all directions, with period $\varepsilon$ ( $\varepsilon$ and $d_{\varepsilon}$ are two positive parameters devoted to tend to zero). We show that the limit behavior of the solutions depends on the limit, $\theta$, of $\varepsilon / d_{\varepsilon}$. Our method permits us to study all the cases simultaneously. For every value of $\theta$ we obtain an homogenized problem where both the macroscopic and microscopic scale appear together. From this problem, we get a strong approximation (corrector) in $\hat{L}^{\hat{L}}\left(\Omega_{\varepsilon}\right)$ of the displacements and the linearized strain tensor. Any isotropic hypothesis is assumed, indeed the unique hypothesis we make on the symmetry of the elasticity tensor is that it transforms the space of symmetric matrices onto itself. The method can also be applied to nonlinear equations and more general structures (bars not crossing completely the structure, plates instead of beams, tall structures, gridworks, etc.), see $/ 7,13 /$.

We finish this section with some bibliographic notes. The more classical method in our knowledge of 
how to deal with reticulated structures involving several small parameters consists in passing to the limit first in a parameter, then in another one, and so on $(/ 2,9-11,14,15 /)$. Note that this procedure implicitly assumes that the first parameter we pass to the limit is much smaller than the second one and so on. It has been successfully applied to diffusion problems where error estimates have been proved. These estimates show that the solution of the limit problem, obtained by passing to the limit in the different parameters successively gives a good approximation of the solutions corresponding to the problems posed on the reticulated structures when the parameters are small enough, but non zero. However, as far as we know, this method has not provided such good results when it has been applied to the elasticity problem, because there are no convergence results proving that if $\varepsilon$ and $d_{\varepsilon}$ are small, then the solution of the elasticity problem is close, in some sense, to the solution of the limit problem obtained by reiteration. Also there are no corrector results. In fact since the limit behavior depends on the ratio of $\varepsilon$ to $d_{\varepsilon}$, the reiterated limit is different according to the chosen order in the parameters (this was proved using the reiterated method in $/ 12 /$ ).

Another approach to this problem is based on the two-scale method with respect to measures $(/ 3-4,16-$ $18 /)$. So in $/ 4 /$, the case $\theta=\lim _{\varepsilon \rightarrow 0}\left(\varepsilon / d_{\varepsilon}\right)=0$ is considered, while in $/ 18 /$ the cases $\theta=0$ and $\theta=+\infty$ are considered (in the last work an additional term is added in the equation which simplifies the problem because it avoids to estimate the Korn constant in $\Omega_{\varepsilon}$ ). In these articles, the case $\theta$ arbitrary is explicitly mentioned as an open problem. Moreover, these papers do not provide a corrector result, i.e., an approximation of the displacements and the linearized strain tensor in the strong topology of $L^{2}$ as we get in this paper.

\section{STATEMENT OF THE PROBLEM. NOTATION AND DEFINITIONS}

For $i \in\{1, \ldots, N\}$ and $\varsigma=\left(\varsigma_{1}, \ldots, \varsigma_{N}\right) \in \mathbf{R}^{N}$, we denote $\varsigma_{i}^{\prime}=\bar{\Sigma}_{m+i} \varsigma_{m} e_{m}$, where $\left\{e_{1}, \ldots, e_{N}\right\}$ is the usual basis in $\mathbf{R}^{N}$.

We set $Y=(-1 / 2,1 / 2)$ and for every $i \in\{1, \ldots, N\}$ we decompose the cube $Y^{N}=J^{\prime}+S^{\prime}$, with $J^{\prime}$ the projection of $Y^{N}$ onto the direction $e_{i}$, i.e., $J^{\prime}=\left\{y_{i} e_{i}: y_{i} \in Y\right\}$, and $S^{\prime}$ the projection of $\gamma^{N}$ onto the orthogonal space to $e_{i}$, i.e., $S^{i}=\left\{y \in Y^{N}: y_{l}=0\right\}$.

For $\varepsilon>0$ and $d_{\varepsilon} \in(0,1)$ converging to zero, we define the open reticulated structure $V_{\varepsilon}$ (see Figures 1 and 2 for the two dimensional case) by

$$
V_{\varepsilon}=\bigcup_{i=1}^{N} V_{\varepsilon}^{i}, \text { with } V_{\varepsilon}^{i}=\bigcup_{k \in \mathbf{Z}^{N}}\left\{x \in \mathbf{R}^{N}: \mid x_{j}-\varepsilon k_{j}<\frac{\varepsilon u_{\varepsilon}^{\prime}}{2}, i \neq i\right\}, i \in\{1, \ldots, N\}, \varepsilon>0
$$

For a smooth bounded open set $\Omega \subset \mathbf{R}^{N}$ we define $\Omega_{\varepsilon}=\Omega \cap V_{\varepsilon}, \Gamma_{\varepsilon}=\bar{\Omega}_{\varepsilon} \cap \partial \Omega$ and $\Omega_{\varepsilon}^{i}=\Omega \cap V_{\varepsilon}^{\prime}, i \in\{1, \ldots, N\}$.

We denote by $H_{\Gamma_{\varepsilon}}^{\dagger}\left(\Omega_{\varepsilon}\right)$ the space of functions in $H^{\prime}\left(\Omega_{\varepsilon}\right)$ which vanish on $\Gamma_{\varepsilon}$. 


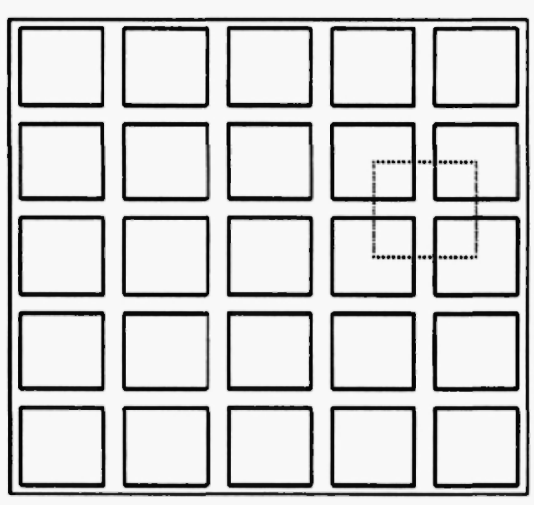

Figure 1.

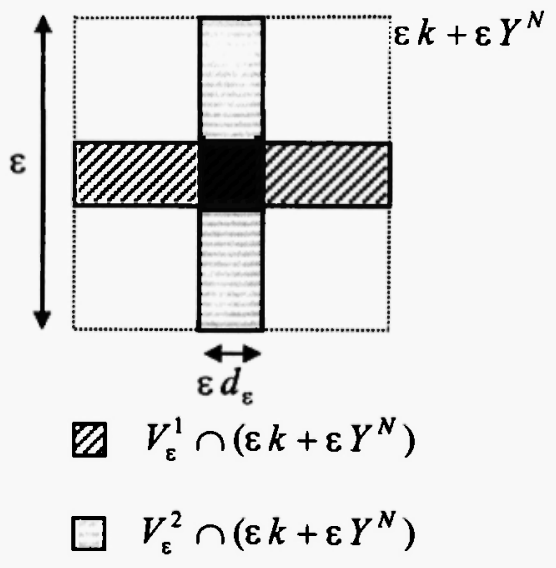

Figure 2.

On $\Omega_{\varepsilon}$ we pose the elasticity problem

$$
\left\{\begin{array}{l}
-\operatorname{div}\left(A e\left(u_{\varepsilon}\right)\right)=F \quad \text { in } \Omega_{\varepsilon} \\
u_{\varepsilon}=0 \text { on } \Gamma_{\varepsilon}, \quad\left(A e\left(u_{\varepsilon}\right)\right) v_{\varepsilon}=0 \text { on } \partial \Omega_{\varepsilon} \backslash \Gamma_{\varepsilon}
\end{array}\right.
$$

where $v_{\varepsilon}$ is the unit outward normal to $\Omega_{\varepsilon}, F \in C^{0}(\bar{\Omega})^{N}$ and $A$ is a definite positive linear operator from the space of $N$-dimensional symmetric matrices onto itself (more general problems are studied in $/ 7 /$ and /13/; see also /6/ for the two-dimensional case). Under these assumptions, (1) admits an unique solution $u_{\varepsilon}$ in $H_{\Gamma_{\varepsilon}}^{1}\left(\Omega_{\varepsilon}\right)^{N}$. Our goal in the present paper is to study the asymptotic behavior of $u_{\varepsilon}$.

To express our result we need some notation and definitions. For a sequence $u_{s}: V_{\varepsilon} \rightarrow \mathbf{R}^{N}$ and $i \in\{1, \ldots, N\}$, we define $\hat{u}_{\varepsilon}^{i}: \mathbf{R}^{N} \times Y^{N} \rightarrow \mathbf{R}^{N}$ by

$$
\hat{u}_{\varepsilon}^{i}(x, y)=\sum_{k \in Z^{N}} u_{\varepsilon}\left(\varepsilon\left(k+y_{i} e_{i}+d_{\varepsilon} y_{i}^{\prime}\right)\right) \chi_{\left(\varepsilon k+\varepsilon Y^{N}\right)}(x), \quad \forall \varepsilon>0
$$

This function will be used to describe the behavior of $u_{\varepsilon}$ in $v_{\varepsilon}^{-i}$. Observe that in $\left(\varepsilon k+\varepsilon Y^{N}\right) \times Y^{N}$, $k \in \mathbf{Z}^{N}, \varepsilon>0, \hat{u}_{\varepsilon}^{i}(x, y)$ does not depend on the macroscopic variable $x$, and as a function of the 
microscopic variable $y$ it is obtained from $u_{\varepsilon}$ by the change of variables

$$
y_{\varepsilon}^{i}(x)=\frac{\bar{x}_{i}-\varepsilon k_{i}}{\varepsilon} e_{i}+\frac{x_{i}^{\prime}-\varepsilon k_{i}^{\prime}}{\varepsilon d_{\varepsilon}}
$$

which transforms $V_{\varepsilon}^{t} \cap\left(\varepsilon k+\varepsilon Y^{N}\right)$ (see Figure 2) into $Y^{N}$.

Denoting by $v$ the unit outward normal to $\Omega$, we define the functional spaces $E^{i}=E_{0}^{i} \times E_{1}^{i} \times E_{2}^{i} \times E_{3}^{i}$, $i \in\{1, \ldots, N\}$, by

$$
\begin{gathered}
E_{0}^{i}=\left\{\hat{u}_{0}^{i} \in L^{2}(\Omega): \partial_{x_{1}} \hat{u}_{0}^{i} \in L^{2}(\Omega), \hat{u}_{0}^{i} v_{i}=0 \text { on } \partial \Omega\right\}, \\
E_{1}^{\prime}=\left\{\dot{u}_{1}^{i} \in L^{2}\left(\Omega ; H^{1}\left(Y^{N}\right)\right)^{N}: \hat{u}_{1}^{i} \text { is } y_{i} \text {-periodic, } \dot{u}_{1 m}^{i}=0 \text { in } L^{2}\left(\Omega \times S^{l}\right),\right. \\
\left.e_{y}\left(\hat{u}_{1}^{i}\right)_{\text {in }}=e_{y}\left(\hat{u}_{1}^{i}\right)_{m n}=0, \quad \forall m, n \in\{1, \ldots, N\} \backslash\{\mathrm{i}\}\right\},
\end{gathered}
$$

$E_{2}^{l}=\left\{\hat{u}_{2}^{l} \in L^{2}\left(\Omega \times Y^{N}\right)^{N}: u_{2, i}^{l} \in L^{2}\left(\Omega \times J^{i}, H^{l}\left(S^{i}\right)\right), \hat{u}_{i, m}^{i} \in L^{2}\left(\Omega ; H^{1}\left(Y^{N}\right)\right), \hat{u}_{2}^{i}\right.$ is $y_{i}$-periodic,

$$
\begin{gathered}
\left.\int_{S^{i}} \hat{u}_{2, m}^{i} d y_{i}^{\prime}=0 \text { in } L^{2}\left(\Omega \times J^{l}\right), \quad e_{y}\left(\tilde{u}_{2}^{i}\right)_{m m}=0, \quad \forall m, n \in\{1, \ldots, N\} \backslash\{\mathrm{i}\}\right\}, \\
E_{3}^{i}=\left\{\hat{u}_{3}^{i} \in L^{2}\left(\Omega \times J^{i} ; H^{1}\left(S^{i}\right)\right)^{N}: \hat{u}_{3, t}^{i}=0\right\} .
\end{gathered}
$$

For $\left(\hat{u}_{0}^{i}, \hat{u}_{1}^{i}, \hat{u}_{2}^{i}, \hat{u}_{3}^{i}\right) \in \bar{L}^{i}$, we define $e_{0}^{i}\left(\hat{u}_{0}^{i}, \dot{u}_{1}^{i}, \hat{u}_{2}^{i}, \dot{u}_{3}^{i}\right) \in L^{2}\left(R^{N} \times Y^{N} ; S^{N}\right)$ by

$$
\begin{gathered}
e_{0}^{i}\left(\hat{u}_{0}^{i}, \dot{u}_{1}^{i}, \hat{u}_{2}^{i}, \hat{u}_{3}^{i}\right)_{i i}=\partial_{x_{1}} \hat{u}_{0}^{i}+\partial_{y_{i}} \dot{u}_{i, i}^{i}, \quad 2 e_{0}^{i}\left(\dot{u}_{0}^{i}, \dot{u}_{1}^{i}, \hat{u}_{2}^{i}, \hat{u}_{3}^{i}\right)_{i n}=\bar{o}_{y_{1}} \hat{u}_{2, n}^{i}+\partial_{y_{n}} \hat{u}_{2, i}^{i} \\
2 e_{0}^{i}\left(\hat{u}_{0}^{i}, \hat{u}_{1}^{i}, \hat{u}_{2}^{i}, \hat{u}_{3}^{i}\right)_{m n}=\partial_{\nu_{m}} \hat{u}_{3, n}^{i}+\partial_{y_{n}} \hat{u}_{3, m}^{i}, \quad \forall m, n \in\{1, \ldots N\} \backslash\{\mathrm{i}\} .
\end{gathered}
$$

\section{ASYMPTOTIC RESULT}

The following theorem provides the asymptotic behavior of the solution of (1).

THEOREM - Let $u_{\varepsilon}$ be the sequence of solutions of (1) and set

$$
\gamma_{\varepsilon}=\frac{1}{\frac{\varepsilon}{d_{\varepsilon}}+1}
$$

We suppose there exists $\lim _{\varepsilon \rightarrow 0} \gamma_{\varepsilon}-\gamma$. (this always holds for a subsequence). We consider a solution $\left(u_{0}^{l}, \dot{u}_{1}^{l}, \hat{u}_{2}^{i}, \hat{u}_{3}^{i}\right) \in E^{t}, i \in\{1, \ldots, N\}$, of the variational problem 


$$
\left\{\begin{array}{l}
\sum_{i=1}^{N} \int_{\Omega \times Y^{N}} A e_{0}^{i}\left(\hat{u}_{0}^{i}, \hat{u}_{1}^{i}, \hat{u}_{2}^{i}, \hat{u}_{3}^{i}\right): e_{0}^{i}\left(\hat{v}_{0}^{i}, \hat{v}_{1}^{i}, \hat{v}_{2}^{i}, \hat{v}_{3}^{i}\right) d y d x=N \gamma \sum_{i=1}^{N} \int_{\Omega \times Y^{N}} F_{i} \hat{v}_{0}^{i} d x+ \\
+(1-\gamma) \sum_{i=1}^{N} \sum_{m \neq i} \int_{\Omega=Y^{N}} F_{m} \dot{v}_{1, m}^{i} d y d x, \quad \forall\left(\hat{v}_{0}^{i}, \hat{v}_{1}^{i}, \hat{v}_{2}^{i}, \hat{v}_{3}^{i}\right) \in E^{i}, i \in\{1, \ldots, N\} .
\end{array}\right.
$$

Then, assuming $\hat{u}_{0}^{i}, \hat{u}_{1}^{i}, e_{0}^{i}\left(\hat{u}_{0}^{i}, \hat{u}_{1}^{i}, \hat{u}_{2}^{i}, \hat{u}_{3}^{i}\right)$ continuous in $\overline{\Omega \times Y^{i i}}$ for $i \in\{1, \ldots, N\}$, the sequences

$$
\begin{aligned}
& g_{\varepsilon, i}^{i}(\cdot)=\frac{1}{\gamma_{\varepsilon}} \hat{u}_{0}^{i}(\cdot), \quad g_{\varepsilon, m}^{i}(\cdot)=\frac{1}{\gamma_{\varepsilon}}\left[\hat{u}_{0}^{m}(\cdot)+\frac{\varepsilon}{d_{\varepsilon}} \hat{u}_{1, m}^{i}\left(\cdot, y_{\varepsilon}^{i}(\cdot)\right)\right], \forall m \neq i, \\
& G_{\varepsilon}^{i}(\cdot)=\frac{1}{\gamma_{\varepsilon}} e_{0}^{i}\left(\hat{u}_{0}^{i}, \hat{u}_{1}^{i}, \hat{u}_{2}^{i}, \hat{u}_{3}^{i}\right)\left(\cdot, y_{\varepsilon}^{i}(\cdot)\right),
\end{aligned}
$$

give the following approximations to $u_{\varepsilon}$ and $e\left(u_{\varepsilon}\right)$

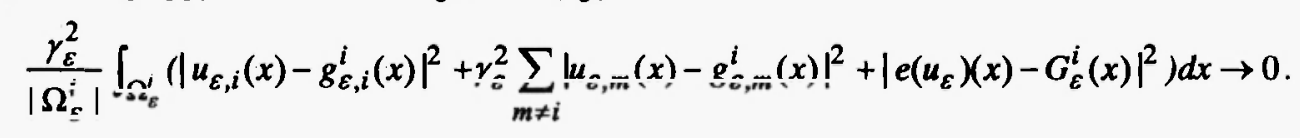

See $/ 7 /$ for the case where the continuity hypotheses on $\hat{u}_{0}^{i}, \hat{\ddot{u}}_{1}^{i}, e_{0}^{i}\left(\hat{u}_{0}^{i}, \hat{u}_{1}^{i}, \hat{u}_{2}^{i}, \hat{u}_{3}^{i}\right)$ does not hold. The proof of the result uses some estimates of Korn's type for the structure considered and a compactness result giving the asymptotic behavior of a sequence $u_{\varepsilon}$ which satisfies

$$
\frac{1}{\left|\Omega_{\varepsilon}\right|} \int_{\Omega_{\varepsilon}}\left|e\left(u_{\varepsilon}\right)\right|^{2} d x<C, \forall \varepsilon>0,
$$

but which is not necessarily the solution of a partial differential problem.

\section{REMARKS AND CONCLUSIONS}

Assuming sufficient smoothness, our result shows that the behavior of $u_{\varepsilon}$ in $\Omega_{\varepsilon}^{i}$ is similar to that of the sequence $z_{\varepsilon}^{\prime}$ defined by

$$
\begin{gathered}
\gamma_{\varepsilon} z_{\varepsilon, i}^{i}=\hat{u}_{0}^{i}+\varepsilon \hat{u}_{1, i}^{i}\left(\cdot, y_{\varepsilon}^{i}\right)+\varepsilon d_{\varepsilon}\left(\hat{u}_{2, i}^{i}\left(\cdot, y_{\varepsilon}^{i}\right)-\sum_{n \neq i}\left(\partial_{x_{n}} \hat{u}_{0}^{i}+\partial_{x_{i}} \hat{u}_{0}^{n}\right) y_{\varepsilon, n}^{i}\right)-\varepsilon^{2} \sum_{n \neq i} \partial_{x_{i}} \hat{u}_{1, n}^{i}\left(\cdot, y_{\varepsilon}^{i}\right) y_{\varepsilon, n}^{i}, \\
\gamma_{\varepsilon} z_{\varepsilon, m}^{i}=\hat{u}_{0}^{r}+\frac{\varepsilon}{d_{\varepsilon}} \hat{u}_{1, m}^{i}\left(\cdot, y_{\varepsilon}^{i}\right)+\varepsilon \hat{u}_{2, m}^{i}\left(\cdot, y_{\varepsilon}^{i}\right)+\varepsilon d_{\varepsilon}\left(\hat{u}_{3, m}^{i}\left(\cdot, y_{\varepsilon}^{i}\right)-\sum_{n \neq l} \partial_{x_{n}} \hat{u}_{0}^{m} y_{\varepsilon, n}^{i}\right)-\varepsilon^{2} \sum_{n \neq l} \partial_{x_{n}} \hat{u}_{1, m}^{i}\left(\cdot, y_{\varepsilon}^{i}\right) y_{\varepsilon, n}^{i},
\end{gathered}
$$

in the following sense

$$
\frac{\gamma_{\varepsilon}^{2}}{\left|\Omega_{\varepsilon}^{i}\right|} \int_{\Omega_{s}^{\prime}}\left(\left|u_{\varepsilon, i}-z_{\varepsilon, i}^{i}\right|^{2}+\gamma_{\varepsilon}^{i} \sum_{m \neq i}\left|u_{\varepsilon, m}-z_{\varepsilon, m}^{i}\right|^{2}+\left|e\left(u_{\varepsilon}-z_{\varepsilon}^{i}\right)\right|^{2}\right) d x \rightarrow 0
$$

Thus, our method does not only give the "limit" of $u_{\varepsilon}$ but it also gives an asymptotic expansion of it. 
If $\theta=\lim _{\varepsilon \rightarrow 0}\left(\varepsilon / d_{\varepsilon}\right)=0$ (i.e., $\left.\gamma=1\right)$ then $\hat{u}_{0}$ defined by $\hat{u}_{0}=\left(\hat{u}_{0}^{1}, \ldots, \hat{u}_{0}^{N}\right)$ is the "limit" of $u_{\varepsilon}$ in the sense

$$
\frac{1}{\left|\Omega_{\varepsilon}\right|} \int_{\Omega_{s}}\left|u_{\varepsilon}(x)-\hat{u}_{0}(x)\right|^{2} d x \rightarrow 0 \text {. }
$$

We point out that the more usual way to study the problem considered here (see $/ 2,11 /)$ is by passing to the limit first in $\varepsilon$ and then in $d_{\varepsilon}$, so it is assumed that $\varepsilon$ is much smaller than $d_{\varepsilon}$. The "limit" obtained by this reiterated procedure coincides with our function $\hat{u}_{0}$.

If $\theta \in(0,+\infty)$ (i.e., $\gamma \in(0,1)$ ), then the function $\hat{u}_{0}=\left(\hat{u}_{0}^{l}, \ldots, \hat{u}_{0}^{l i}\right)$ is no longer the limit of $u_{\varepsilon}$. In fact if we look for a "weak limit" $u$ of $u_{\varepsilon}$ defined by (see $/ 4,18$ / where this type of limit is considered)

$$
\frac{1}{\left|\Omega_{\varepsilon}\right|} \int_{\Omega_{r}} u_{\varepsilon}(x) \varphi(x) d x \rightarrow \frac{1}{|\Omega|} \int_{\Omega} u(x) \varphi(x) d x, \forall \varphi \in C_{0}^{j}(\Omega)^{N}
$$

our result shows that this limit $u$ of $u_{\varepsilon}$ is given by

$$
u(x)=\frac{1}{\gamma} \hat{u}_{0}(x)+\frac{1-\gamma}{N \gamma^{2}} \sum_{j=1}^{N} \sum_{m \neq j} j_{Y^{N}} \hat{u}_{1, j}^{m}(x, y) d y e_{j} \text {, a.e. } x \in \Omega
$$

Finally, if $\theta=+\infty$ (i.e., $\gamma=0$ ) the deformations $u_{\varepsilon}$ and the linearized strain tensor $e\left(u_{\varepsilon}\right)$ tend to infinity (then the linearized elasticity model, which assumes small deformations, can be inadequate if $d_{\varepsilon}$ is much smaller than $\varepsilon$ ). In this case what we have is that $\gamma_{\varepsilon}^{2} u_{\varepsilon}$ converges in the sense (3) to $w$ defined by

$$
w(x)=\lim _{\varepsilon \rightarrow 0} \gamma_{\varepsilon}^{2} u_{\varepsilon}=\frac{i}{N^{\prime}} \sum_{j=1}^{N} \sum_{m \neq j} \int_{\gamma^{N}} \hat{u}_{1, j}^{m}(x, y) d y e_{j} \text {, a.e. } x \in \Omega
$$

Observe that $w$ does not depend on $\hat{u}_{0}$; it only depends on the functions $\hat{u}_{l, J}^{m}$.

In the case where $A$ is given by $A E=\lambda \operatorname{trace}(E) I+2 \mu E$, for every symmetric matrix $E$, with $\lambda>0$, $\mu>0$ (isotropic material), we deduce from (2) that $\hat{u}_{0}=\left(\hat{u}_{0}^{1}, \ldots, \hat{u}_{0}^{N}\right)$ (which as we said above is the "limit" of $u_{\varepsilon}$ when $\varepsilon / d_{\varepsilon}$ tends to zero) satisfies the degenerated problem

$$
-\Lambda \partial_{x_{i} x_{i}}^{2} \hat{u}_{0}^{i}=N \gamma F_{:} \quad \text { in } \Omega, i \in\{1, \ldots, N\}, \quad \text { with } \Lambda=\frac{2 \mu(\lambda N+2 \mu)}{\lambda(N-1)+2 \mu}
$$

To obtain an elliptic problem, some authors (see $/ 2 /, / 8 /$ ) propose introducing additional bars in the structure (reinforced structures). Passing to the limit first in $\varepsilon$ and then in $d_{\varepsilon}$ (which implies $\varepsilon$ is much smaller than $d_{\varepsilon}$, or cquivalently $\theta=0$ ) they obtain a non-degenerated problem for the limit $\hat{u}_{0}$ of $u_{\varepsilon}$. 
However, our method applied to these structures with additional bars (see $/ 7 /, / 13 /$ ) shows that the limit behavior of $u_{\varepsilon}$ still depends on the limit $\theta$ of $\varepsilon / d_{\varepsilon}$. Although we prove that the corresponding function $\hat{u}_{0}$ we obtain in this case satisfies a non-degenerated problem for any value of $\theta$, we emphasize that $\hat{u}_{0}$ is not in general the limit of $u_{\varepsilon}$. It only gives the limit of $u_{\varepsilon}$ when $\theta=0$, which corresponds in fact with the case studied in the mentioned papers $/ 2 /, / 8 /$. Even adding additional bars, we prove in $/ 7 /$ that the problem satisfied for the limit of $u_{\varepsilon}$ in the sense (3) is degenerated when $\theta \neq 0$.

\section{ACKNOWLEDGEMENTS.}

The authors wish to thank F. Murat for stimulating discussions on the topic. This work has been partially supported by the project BFM2002-00672 of the Ministerio de Ciencia y Tecnologia of Spain.

\section{REFERENCES}

1. T. Arbogast, J. Douglas and U. Hornung, Derivation of the double porosity model of single phase flow via homogenization theory, SIAM.J. Math. Anal., 21, 823-836 (1990).

2. N.S. Bakhvalov and G.P. Panasenko, Homogenization: Averaging Processes in Periodic Media, Math. Appl , Kluwer Academic Publishers, Vol. 36, Dordrecht, 1989.

3. G. Bouchitté and I. Fragalá, Homogenization of thin structures by two-scale method with respect to measures, SIAMIJ. Math. Anal, 32 (6), 1198-1226 (2001).

4. G. Bouchitté and I. Fragalá, Homogenization of elastic thin structures: a measure-fattening approach, $J$. Convex Anal. 2, 339-362 (2002).

5. J. Casado-Diaz, M. Luna-Laynez and J.D. Martin, An adaptation of the multi-scale methods for the analysis of very thin reticulated structures, C.R. Acad. Sci. Paris, Sér. I, 332 (3), 223-228 (2001).

6. J. Casado-Dlaz and M. Luna-Laynez, A multiscale method to the homogenization of elastic thin reticulates structures, in: Homogenization 2001. Proceedings of the First HMS2000 International School and Conference on Homogenization, L. Carbone and R. De Arcangelis (Eds.), GAKUTO Internat. Ser. Math. Sci. and Appl., 18, Gakkotosho, Tokyo, 2003; pp. 155-168.

7. J. Casado-Díaz and M. Luna-Laynez, Homogenization of the anisotropic, heterogeneous, linearized elasticity system in thin reticulated structures, Proc. R. Soc. Edinb. A, 134, $1041-1083$ (2004).

8. R. Chineb, D. Cioranescu, A. El Jatani and G. Panasenko, Structures réticulées reforcées en élasticité, C.R. Acad. Sci. Paris, Sér. I, 326 (3), 897-902 (1998).

9. D. Cioranescu and J. Saint Jean Paluin, Reinforced and honeycomb structures, J. Math. Pur. Appl., 65, 403-422 (1986).

10. D. Cioranescu and J. Saint Jean Paluin, Problèmes de Neumann et Dirichlet dans des structures 
reticulees de faible epaisseur, C.R. Acad. Sci. Paris, Sér. I, 303 (1), 7-12 (1986).

11. D. Cioranescu and J. Saint Jean Paluin, Homogenization of Reticulated Structures, Appl. Math. Sci., Vol. 136, Springer-Verlag, New York, 1999.

12. G. Griso, Analyse asymptotique de structures réticulées, Thèse de l'Universite Pierre et Marie Curie (Paris Vl), 1996.

13. M. Luna-Laynez, Homogeneización de estructuras reticuladas: un metodo multiescala, Ph.D. Thesis, University of Seville, 2003.

14. G.P. Panasenko, The principle of splitting of an averaged operator for a nonlinear system of equations in periodic and random skeletal structures, Soviet Math. Dokl., 25 (2), 290-295 (1982).

15. G.P. Panasenko, Averaging processes in frame constructions with random properties, Vych. Mat. Fiz., 23 (5), 1098-1109 (1983).

16. V.V. Zhikov, Connectedness and homogenization. Examples of fractal conductivity, Mat. Sb., 187 (7), 3-40 (1996).

17. V.V. Zhikov, On an extension of the method of two-scale convergence and its applications, Mat. Sb., 191 (7), 973-1014 (2000).

18. V.V. Zhikov, Homogenization of elasticity problems on singular structures, Dokl. Math., 64 (2), 258262 (2001). 